\title{
Collectin-11: a signal for complement activation
}

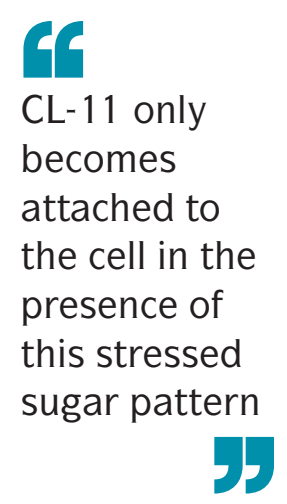

Activation of complement on the cell surface is sufficient to induce cell injury leading to inflammation and cell death; however, the initiation signal that triggers complement activation in response to injury is unknown. Now, Steven Sacks and colleagues report that the soluble C-type lectin, collectin-11 (CL-11), is released from tubule cells and in the presence of cell stress, binds L-fucose to initiate complement activation on the cell surface. "Our study identifies an important link between ischaemic or hypoxic stress and complement-mediated renal cell injury," explains Sacks. “Our findings also represent a promising step towards identifying approaches to prevent morbidity and mortality in acute kidney injury (AKI)."

Previous work demonstrating that CL-11 can activate complement on pathogen surfaces by recognizing sugar molecules prompted Sacks and colleagues to assess the ability of CL-11 to trigger complement activation on renal cells under stress, and whether this trigger could be induced through the recognition of fucose - a common component of the extracellular matrix. The researchers found that ischaemia-reperfusion injury increased the intrarenal expression of CL-11, which co-localized with fucose and the complement activation

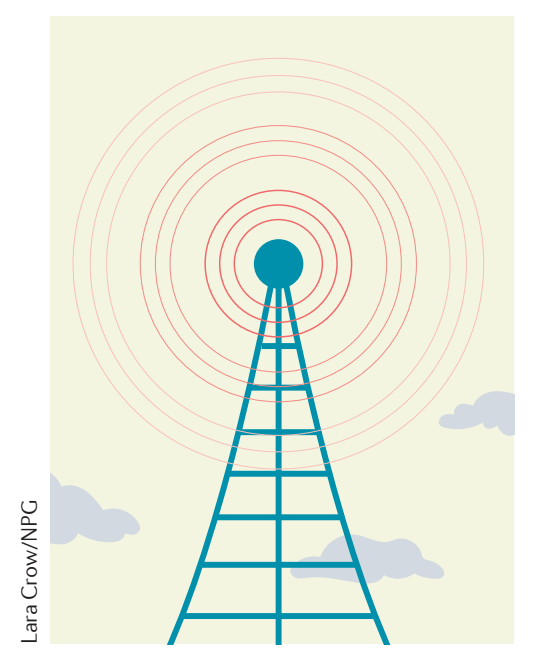

product, C3d, in the proximal tubule. Transplantation of kidneys from CL-11-deficient mice, but not control mice, protected the transplant recipient against $\mathrm{AKI}$ induced by ischaemia-reperfusion, suggesting a direct role of kidney-derived CL-11 in inducing renal injury. "Our transplantation studies were important in understanding the biological relevance of our in vitro findings," explains Sacks. "Our studies using cultured mouse and human kidney tubule cells demonstrated that following cell stress - induced by low oxygen tension or hypothermia - an abnormal pattern of fucose presents on the cell surface, which provides an anchor point for CL-11 attachment and subsequent deposition of complement."

Sacks and colleagues believe that their research highlights two important findings. "The first is that distressed mammalian renal tubule cells, following exposure to ischaemia or hypoxia, display an aberrant staining pattern of fucose on the cell surface," explains Sacks. "The second point of interest is that CL-11 only becomes attached to the cell in the presence of this stressed sugar pattern. Only where the altered pattern of fucose and CL-11 come together does complement activation occur."

The researchers hope their findings might inform the development of treatment strategies to prevent ischaemic damage in AKI or kidney transplantation. "Treatments to inhibit complement activation are currently in clinical trials, but this work identifies a higher step in the injury pathway in which preventative action might be taken," says Sacks. "We need to understand more about the changes in fucose presentation on the cell surface and how the interaction with CL-11 can be exploited for clinical benefit."

Susan J. Allison

ORIGINAL ARTICLE(S) Farrar, C. A. et al. Collectin-11 detects stress-induced L-fucose pattern to trigger renal epithelial injury. J. Clin. Invest. http://dx.doi.org/10.1172/JCl83000 